\section{"Bomba hormonal": os riscos da contracepção de emergência na perspectiva dos balconistas de farmácias no Rio de Janeiro, Brasil}

\author{
"Hormone bomb": risks of emergency \\ contraception from the perspective of pharmacy \\ attendants in Rio de Janeiro, Brazil
}

\author{
"Bomba hormonal": los riesgos de la anticoncepción \\ de emergencia desde la perspectiva de los \\ auxiliares de farmacias en Río de Janeiro, \\ Brasil
}

\section{Resumo}

A pesquisa objetivou conhecer a perspectiva dos balconistas de farmácias sobre a contracepção de emergência na Região Metropolitana do Rio de Janeiro, Brasil. O material empírico advém de pesquisa socioantropológica com vinte entrevistas semiestruturadas com balconistas dos sexos feminino (8) e masculino (12). Os entrevistados apresentam concepções negativas sobre a contracepção de emergência, enfatizando os riscos que ela pode provocar à saúde. O medicamento é considerado uma "bomba hormonal" que pode causar danos aos órgãos reprodutivos femininos e outros sistemas do corpo. Eles destacam os riscos do uso "descontrolado" ou "indiscriminado", especialmente por adolescentes e mulheres jovens. Por ser considerado "perigoso" aos corpos femininos, eles atribuem a responsabilidade de orientação e aconselhamento sobre o uso do método aos médicos ginecologistas e não aos farmacêuticos. Discute-se a necessidade de ampliação do debate público sobre contracepção de emergência no Brasil, incluindo-se os farmacêuticos e balconistas de farmácia, além dos profissionais de saúde e educadores.

Anticoncepção Pós-Coito; Atenção Farmacêutica;

Sexualidade; Saúde Reprodutiva
Elaine Reis Brandão 1

Cristiane da Silva Cabral 2

Miriam Ventura 1

Sabrina Pereira Paiva 3

Luiza Lena Bastos 1

Naira Villas Boas Vidal de Oliveira 4

Iolanda Szabo 1

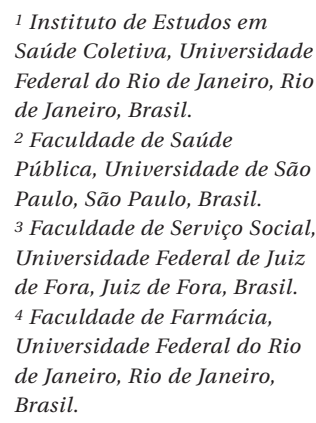

1 Instituto de Estudos em Saúde Coletiva, Universidade Federal do Rio de Janeiro, Rio de Janeiro, Brasil.

2 Faculdade de Saúde Pública, Universidade de São Paulo, São Paulo, Brasil. ${ }^{3}$ Faculdade de Serviço Social Universidade Federal de Juiz de Fora, Juiz de Fora, Brasil. ${ }_{4}^{4}$ Faculdade de Farmácia. Universidade Federal do Rio de Janeiro, Rio de Janeiro, Brasil.

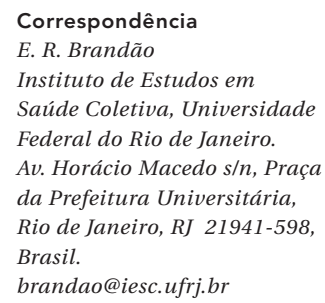




\section{Introdução}

Este estudo teve por objetivo conhecer a visão dos balconistas de farmácias da Região Metropolitana do Rio de Janeiro sobre a comercialização da contracepção de emergência, buscando compreender as suas representações sobre o medicamento e o que pensam acerca do uso deste método pelas consumidoras que atendem no seu local de trabalho.

A contracepção de emergência ou "pílula do dia seguinte", como é designada no senso comum, é um contraceptivo que pode ser utilizado em situações emergenciais, após a relação sexual desprotegida, devendo ser administrado dentro do prazo de 120 horas para se evitar uma gravidez. É um método contraceptivo importante a ser ofertado pelas políticas públicas de planejamento reprodutivo para a garantia da autonomia reprodutiva feminina.

Analisando os dados da última Pesquisa $\mathrm{Na}$ cional de Demografia e Saúde da Criança e da Mulher (PNDS), de 2006, Perpétuo \& Wong ${ }^{1}$ ressaltam uma ampliação do uso de anticoncepcionais no país em relação à pesquisa anterior de 1996, com redução significativa do recurso à laqueadura tubária, entre mulheres de 15 a 44 anos, em união conjugal. No entanto, a esterilização feminina $(25,9 \%)$ e a pílula oral $(27,4 \%)$ continuam sendo os dois métodos mais prevalentes na população. Um percentual de $18,4 \%$ de mulheres desse segmento declarou não usar método algum para prevenir a gravidez.

A oferta de métodos contraceptivos na rede pública de serviços de saúde não contempla a demanda existente, havendo um grande número de pessoas que se dirige às farmácias para comprar a pílula anticoncepcional de uso diário ou o contraceptivo de emergência, com preço médio aproximado de $\mathrm{R} \$ 20,00$, sem necessariamente receber orientação prévia. Segundo a PNDS 2006 2, as farmácias aparecem como fonte de obtenção de métodos contraceptivos modernos para $42,5 \%$ das usuárias de 15 a 49 anos, reunindo os métodos hormonais (pílula e injeção) e preservativo masculino. Tal via de acesso representa uma alternativa importante, já que os estabelecimentos farmacêuticos permanecem abertos à noite e fins de semana, facilitando a obtenção do contraceptivo em tempo oportuno, e contam com farmacêuticos para atendimento aos consumidores. Embora haja recomendação da Agência Nacional de Vigilância Sanitária (ANVISA) de que a venda do contraceptivo de emergência seja feita mediante prescrição médica, tal norma não é respeitada no país. A prática da automedicação na população brasileira é significativa 3,4 , e um estudo recente de revisão siste- mática sobre a prevalência da automedicação na população adulta 5 apontou taxa de $35 \%$ em três trabalhos selecionados com período recordatório de 15 dias.

Segundo a legislação vigente, os estabelecimentos farmacêuticos têm o dever legal de zelar pela manutenção da qualidade e segurança de produtos ofertados, bem como pelo uso racional de medicamentos, a fim de evitar riscos e efeitos nocivos à saúde 6 . No entanto, as farmácias ocupam uma posição ambígua no sistema de saúde brasileiro, na medida em que têm uma dimensão de interesse público ao incidirem sobre práticas de cuidado à saúde, mas são regidas pela lógica privada do mercado de produtos farmacêuticos 7,8 .

$\mathrm{Na}$ literatura sobre contracepção de emergência no Brasil 9 não há estudos acerca da sua dispensação farmacêutica. O processo de dispensação de medicamentos nas farmácias está sob a responsabilidade do farmacêutico. No entanto, na prática cotidiana do comércio farmacêutico no país, o balconista é, em geral, o funcionário responsável por vender os medicamentos, mantendo contato mais estreito com os consumidores. O balconista é, em sua maioria, um profissional com escolaridade até o Ensino Médio, sem conhecimentos específicos sobre o uso de medicamentos, a não ser pelo contato com o material de divulgação dos laboratórios farmacêuticos. A penetração dos processos de medicalização e de "farmacologização" da sociedade 10,11 ampliam cada vez mais as relações diretas entre indústria farmacêutica e seus consumidores, profissionais de saúde e vendedores.

Investigações anteriores sobre a contracepção de emergência apontam expressivas barreiras e dificuldades de implementação das políticas públicas de distribuição desta contracepção $12,13,14,15,16$. Há relatos de preconceitos, discriminação, desinformação e interferência de valores morais e religiosos nas práticas de profissionais de saúde e gestores públicos que recriminam o uso do contraceptivo de emergência por considerá-lo abortivo ou por não aprovarem o livre exercício sexual das mulheres como um direito, subjugando-o ao casamento. Tendo em vista os constrangimentos e obstáculos que as mulheres enfrentam nos serviços públicos de saúde para acesso à contracepção de emergência, o estudo buscou conhecer os valores e sentidos associados ao método por quem o dispensa nas farmácias. Discutir as representações sociais dos profissionais de farmácia a respeito da contracepção de emergência pode esclarecer concepções sobre sexualidade feminina, corpo e reprodução que estão influenciando as suas práticas junto à população usuária. 


\section{Procedimentos metodológicos}

A pesquisa Uma Investigação Socioantropológica em Farmácias: Posição de Farmacêuticos e Balconistas sobre a Contracepção de Emergência, desenvolvida em 2012-2014, buscou conhecer as perspectivas de profissionais que trabalham em farmácias sobre a contracepção de emergência. A questão de pesquisa que ensejou tal estudo foi a necessidade de conhecer as circunstâncias sociais que condicionam o acesso das mulheres à contracepção de emergência nas farmácias privadas, mediado pela interação com farmacêuticos e balconistas. Já se conhece as muitas dificuldades que cercam o acesso das mulheres ao método na rede pública de serviços de saúde, em razão de preconceitos e juízos de valor dos profissionais de saúde sobre as práticas sexuais femininas. Tendo em vista a busca maciça da contracepção de emergência no comércio farmacêutico, intencionávamos saber se os mesmos constrangimentos existiam naquele espaço, como se dava tal interação, como as representações sociais destes profissionais se refletem em suas práticas profissionais.

A pesquisa contou com estratégias diferenciadas para coleta de dados entre as duas categorias de trabalhadores de farmácia: um questionário fechado, estruturado, autoaplicado, anônimo, para os farmacêuticos $(n=383)$ e entrevistas face a face com roteiro semiestruturado de questões com vinte balconistas da Região Metropolitana da cidade do Rio de Janeiro, Brasil, 12 homens e 8 mulheres. A proposta deste artigo é discutir os resultados do estudo qualitativo com os balconistas.

As entrevistas com os balconistas ocorreram entre agosto de 2012 e maio de 2013. Foram realizadas por três pesquisadoras com formação em Ciências Sociais, treinamento nos temas da sexualidade, gênero, saúde reprodutiva e contracepção, para conseguirem abordá-los com naturalidade junto aos sujeitos. O roteiro da entrevista foi organizado em quatro módulos: (1) perfil sociodemográfico dos entrevistados; (2) venda da contracepção de emergência e interação social com consumidoras no balcão da farmácia; (3) opinião sobre contracepção de emergência e sua regulação sanitária no Brasil; (4) experiência de uso pessoal da contracepção de emergência.

Para a seleção dos balconistas, adotou-se inicialmente uma estratégia de rede de indicações 17 , em que o primeiro balconista entrevistado indica outro para ser contatado pela equipe de pesquisa, preferencialmente de farmácia e região distintas, para se captar a diversidade de experiências sociais e profissionais, e assim sucessivamente. Entretanto, houve dificuldades para se obter a totalidade dos vinte entrevistados por essa estratégia de captação, devido à indisponibilidade de tempo para entrevista entre os sujeitos contatados. Em geral, eles têm uma jornada de trabalho superior a 40 horas semanais, além de muitos se dedicarem aos estudos nos horários noturnos ou quando não estão no local de trabalho. Assim, no trabalho de campo, também foi adotada uma abordagem mais direta, com visitas das pesquisadoras a diferentes estabelecimentos farmacêuticos para contato direto e convite à participação na pesquisa.

As entrevistas foram gravadas com anuência dos sujeitos, mediante Termo de Consentimento Livre e Esclarecido. Foram realizadas no espaço da própria farmácia (13), devido à indisponibilidade dos balconistas se ausentarem do trabalho durante o expediente e de conseguirem horários extras para as entrevistas; ou em suas proximidades (7), como em parques ao ar livre, cafés e lanchonetes, antes da entrada ou após a saída dos entrevistados dos seus respectivos locais de trabalho. Embora algumas entrevistas tenham sido realizadas durante o expediente dos informantes, a maioria não demonstrou receio ou intimidação, tornando-a mais uma "conversa de balcão".

A análise dos dados coletados baseou-se na transcrição literal das entrevistas, sem identificação nominal dos participantes, para preservar o sigilo, a confidencialidade e o anonimato dos sujeitos. Um diário de campo das circunstâncias sociais que cercaram a entrevista, desde o contato inicial com o entrevistado, por intermédio de indicação de amigos ou por visita à farmácia, até a realização do encontro, as reações e atitudes dos participantes durante a conversa com as pesquisadoras, todas as observações foram registradas para serem somadas aos discursos nativos dos balconistas. Houve leitura intensiva e repetitiva das entrevistas transcritas e diários de campo, cotejando-os com estudo etnográfico prévio em farmácia do Rio de Janeiro 18. Buscou-se captar os elementos recorrentes e relevantes nos discursos dos entrevistados, para se extrair as categorias temáticas mais expressivas e significativas da visão de mundo dos participantes $19,20,21,22$.

As narrativas dos entrevistados trazem mais semelhanças do que discordâncias entre eles. Embora se saiba que diferenças de gênero possam determinar concepções distintas sobre um fato social, não foram observadas interferências significativas nas representações dos pesquisados segundo o sexo ou tempo de atuação no mercado farmacêutico. Pode-se dizer que balconistas homens e mulheres compartilham uma visão moralista e condenatória à prática de uso 
da contracepção de emergência. Assim, foi possível constatar vários pontos em comum com os resultados de Paiva 18 no que se refere a um certo ethos do balconista, sua maneira peculiar de pensar e atuar em relação ao público atendido. Alguns temas se tornaram estratégicos para a discussão dos resultados: os riscos que a contracepção de emergência provoca à saúde das mulheres e a regulação pública de sua dispensação nas farmácias.

O projeto de pesquisa foi aprovado pelo Comitê de Ética em Pesquisa do Instituto de Estudos em Saúde Coletiva, Universidade Federal do Rio de Janeiro (IESC/UFRJ), em 03 de outubro de 2012, com parecer no 114.610 (CAAE: 04918212.9.0000.5286).

\section{Resultados e discussão}

\section{Perfil sociodemográfico dos balconistas}

Do total de vinte balconistas entrevistados, oito são do sexo feminino e 12 do masculino. A faixa etária varia de 20 anos (mais jovem) a 63 (mais velho) entre homens e mulheres. A média de idade para as mulheres foi de 28 anos, e para os homens de 39. A escolaridade dos informantes oscila entre o Ensino Médio (um tem o Ensino Médio incompleto e nove completaram este ciclo) e o Ensino Superior (seis declaram ter nível superior incompleto, um o superior completo e um tem pós-graduação). Algumas informações não puderam ser obtidas para dois entrevistados.

Os participantes trabalhavam em farmácias de três municípios adjacentes, Rio de Janeiro, Niterói e São Gonçalo, em bairros com população de diferentes estratos socioeconômicos, em estabelecimentos comerciais distintos. A seleção dos locais de pesquisa obedeceu ao critério de diversificação dos espaços socioeconômicos e culturais para captação dos entrevistados, pois buscava-se uma variação entre farmácias situadas em regiões/zonas sul, norte e centrais das cidades. Os estabelecimentos comerciais integravam 12 grandes redes de farmácias presentes na região.

Mais da metade dos entrevistados se autodenominaram pardos (11), quatro se consideraram pretos e três de cor branca. Sobre filiação religiosa, houve uma predominância de pesquisados de religiões católica (sete) e evangélica (sete), em suas várias vertentes pentecostais; seis declararam não frequentar religião alguma.

Nenhuma das mulheres entrevistadas tem filhos. Elas são mulheres jovens, entre 20 e 33 anos, quatro delas solteiras e outras quatro casadas. Com relação à situação conjugal e número de filhos dos 12 entrevistados homens, oito são casa- dos, entre estes somente um não tem filhos; um é divorciado com filhos; e um solteiro e sem filhos.

A renda média individual mensal declarada entre balconistas mulheres foi de aproximadamente R\$ $1.200,00$ e entre os homens de R\$ $2.000,00$, revelando que as mulheres percebem rendimentos inferiores aos homens ocupando o mesmo cargo, em razão de discriminação de gênero e menor tempo de atuação no ramo farmacêutico.

Quanto ao tempo de trabalho no comércio farmacêutico, a média de anos varia conforme o sexo. Entre mulheres, elas atuam em média há aproximadamente quatro anos em farmácias, e entre os homens há aproximadamente 19 anos. Durante o período em que atuaram em farmácias, alguns deles (homens e mulheres) exerceram outros cargos anteriores à função de balconistas: operador de caixa registradora (oito), perfumista (dois), auxiliar de serviços gerais (dois), gerente (um), entregador (três), estoquista (três). Quanto ao tempo de trabalho que têm na farmácia atual, dentre as oito mulheres, cinco delas atuam no mesmo local desde que ingressaram no comércio farmacêutico e três outras variavam entre 3 meses e 4 anos. Entre os homens, eles têm maior mobilidade em diferentes farmácias ao longo da carreira. O tempo médio de atuação na farmácia atual é de 5 anos.

\section{Os perigos da contracepção de emergência}

Os balconistas entrevistados apresentam sérias preocupações em relação aos efeitos da contracepção de emergência no organismo feminino e quanto à forma como percebem que este medicamento está sendo consumido. Em suas narrativas é dominante a compreensão de que a contracepção de emergência é um "medicamento perigoso" para a saúde das mulheres, pelos inúmeros riscos que apresenta. Ela é por eles considerada como uma "bomba hormonal", em alusão aos seus efeitos explosivos e destrutivos aos corpos femininos, pelos danos que poderia provocar em suas usuárias e em seus futuros bebês.

Para eles, o poder de ação dos hormônios no organismo pode ser arrasador. Considerado importante para o corpo, quando são utilizados em excesso podem causar desequilíbrio orgânico, destruindo não somente os órgãos sexuais e reprodutivos, mas também outras partes do corpo como ossos, podendo causar desde distúrbios de comportamento até dores de cabeça. As percepções sobre os riscos da contracepção de emergência estão ligadas à crença de que este é um medicamento "mais forte" e "perigoso", daí a menção ao termo "bomba hormonal". Eles avaliam como "mais forte" porque consideram 
que a contracepção de emergência contém alta dosagem de hormônios, se comparada às pílulas hormonais orais de uso contínuo, que o grupo não reprova. E "perigoso” porque esses riscos à saúde podem ser multiplicados com o uso indevido pelas mulheres, de forma repetitiva e não emergencial, o que consideram um uso "banalizado”, corriqueiro do medicamento, contrário à sua indicação.

O receio que os entrevistados expressam está ligado à representação de que o medicamento contém altas dosagens hormonais. Essa concepção integra representações sociais mais amplas disseminadas sobre a contracepção de emergência nos contextos internacional e nacional, sendo possível observá-la em vários estudos com usuários e provedores dos serviços de saúde 23,24,25,26,27.

Os pesquisados defendem que os anticoncepcionais de uso diário têm uma dosagem hormonal mais baixa e por isto menos deletéria para a saúde das mulheres. Os anticoncepcionais de uso rotineiro, segundo os entrevistados, são utilizados, em geral, por mulheres mais velhas e casadas, as quais não sentem constrangimento em comprá-los com o balconista da farmácia, independentemente deste ser homem ou mulher. Revelam que a pílula hormonal de uso contínuo é vendida com regularidade em todos os dias da semana e estão entre os produtos mais consumidos nas farmácias.

Em contraste, consideram que a contracepção de emergência tem sido utilizada por mulheres mais jovens e solteiras, com vendas mais expressivas nos fins de semana e feriados. Há uma apreciação distinta sobre dois tipos de contraceptivos hormonais usados pelas mulheres - o de uso rotineiro e o emergencial. Por que o primeiro medicamento não é considerado "perigoso" e capaz de trazer riscos à saúde tal como o segundo? Parece que a "irresponsabilidade" feminina, ao não se proteger antes da relação sexual, está em questão na aquisição e uso da contracepção de emergência. O uso da contracepção regular traz implícito a ideia de "corpos disciplinados", de regulação dos corpos, enquanto a contracepção de emergência sugere a indisciplina e o descontrole do sexo e dos corpos 28 . Há uma reprovação moral daquelas mulheres, em especial, as mais jovens, que se lançam ao prazer sexual sem as precauções devidas, consideradas mulheres "livres".

Em um estudo francês na África Ocidental 29 , tais representações negativas quanto aos efeitos dos "hormônios" no organismo feminino também apareceram, especialmente por parte das mulheres entrevistadas, que demonstraram medo de ficarem estéreis devido à possibilidade do "excesso de hormônios" prejudicar seu organismo. No contexto dos países africanos, as representações relativas ao perigo desses medicamentos se referem a todos os anticonceptivos e não somente à contracepção de emergência. Entretanto, a contracepção de emergência é menos temida do que a contracepção hormonal de rotina, por ser tomada somente em algumas ocasiões e não diariamente.

Além do anticoncepcional oral hormonal de uso rotineiro, eles foram consultados sobre as situações de venda de preservativos masculinos e de medicamentos para tratamento de doenças sexualmente transmissíveis (DST) e disfunção erétil. Os balconistas expressam receio quanto ao abandono do preservativo masculino para prevenção das DST. De acordo com um dos entrevistados, os consumidores não se preocupam com a prevenção de DST e preferem arriscar com a "pílula do dia seguinte". Para eles, o medicamento é "perigoso" também porque provoca o relaxamento de condutas preventivas à gravidez e às DST, ao transmitir a ideia de uma relativa segurança, provocada pela existência de um método anticonceptivo pós-coito. Assim, comportamentos "de risco" poderiam se multiplicar.

Entre os medicamentos relacionados à sexualidade, aquele que mais os leva a comparações com a venda da contracepção de emergência é o citrato de sildenafil, difundido na década de 1990 para o tratamento de disfunção erétil. No Brasil, não há exigência de prescrição médica para a comercialização desse produto nas farmácias. De acordo com os pesquisados, esse é um dos medicamentos mais vendidos atualmente, sendo que um balconista explicou que ele não era vendido atrás do balcão das farmácias (behind-the-counter), mas fica exposto nas gôndolas para o livre acesso do cliente, o que pode facilitar ainda mais a sua aquisição.

Segundos os entrevistados, tal como a venda da contracepção de emergência, os maiores consumidores dessa classe de medicamentos são também os jovens, que costumam demonstrar constrangimento ao comprá-lo no balcão, assim como as consumidoras da contracepção de emergência. $\mathrm{O}$ sentimento de vergonha é ampliado quando não há coincidência entre o sexo/ gênero do balconista que atende o consumidor no balcão da farmácia e aquele que compra o medicamento. Em geral, eles afirmam que as consumidoras preferem comprar a contracepção de emergência no balcão da farmácia com as balconistas, e os consumidores homens preferem comprar o medicamento para melhorar a sua performance sexual com os balconistas. Há na acepção dos balconistas, respaldada por estudos feitos sobre esse medicamento no Brasil e América Latina $30,31,32$, um uso recreativo do citrato 
de sildenafil, para aperfeiçoamento do desempenho sexual masculino juvenil.

$\mathrm{Na}$ analogia entre o consumo da contracepção de emergência e o do medicamento para disfunção erétil masculina, o que se destaca é a ausência do discurso contestatório à venda do citrato de sildenafil por parte dos entrevistados, desconsiderando os possíveis riscos que o mesmo pode representar para a saúde dos homens. Por que então as reservas surgem de modo veemente sobre a venda e uso da contracepção de emergência e suas consequências deletérias para a saúde das mulheres? A reiteração de uma forte divisão de gênero na apreciação moral que fazem entre a dedicação dos homens ao sexo e das mulheres à reprodução, tornaria positiva a preocupação masculina de melhoria do desempenho no ato sexual e negativa a preocupação feminina tardia com a possível ocorrência de uma gravidez. Assim, as razões do uso da contracepção de emergência ser tão criticado entre eles tornamse mais claras.

\section{A contracepção de emergência e sua regulação sanitária no Brasil}

No debate científico e político internacional a respeito da ampliação do acesso das mulheres à contracepção de emergência, um dos temas centrais tem sido a recente proposta de liberação da venda do medicamento over-the-counter (OTC), ou seja, disponível nas gôndolas, para livre acesso do cliente ${ }^{9}$. Está em discussão a possibilidade de ampliação de sua dispensação nas farmácias, regida por protocolos públicos de cooperação mútua entre farmacêuticos e médicos, com o aconselhamento em saúde sexual e reprodutiva pelos farmacêuticos às mulheres no momento da compra da contracepção de emergência. No Brasil, as condições de acesso ao método são radicalmente distintas. Sua distribuição pública no Sistema Único de Saúde (SUS) estabelece sua dispensação por médicos ou enfermeiros nos centros de atenção básica à saúde e unidades do Programa Saúde da Família, além dos programas de atenção às vítimas de violência sexual, existentes em hospitais públicos do país. No entanto, tal distribuição não consegue atender as necessidades da população, com provisão irregular, havendo dificuldades para se obter gratuitamente o medicamento. Assim, grande parte da população brasileira adquire esse medicamento comprando -o nas farmácias, diretamente no balcão (behindthe-counter), com o balconista, sem orientação ou aconselhamento do farmacêutico, pois as políticas públicas de distribuição da contracepção de emergência no Brasil não incluem as farmácias como local de dispensação do método.
Considerando tal contexto, os balconistas foram consultados a respeito da venda desse medicamento nas gôndolas (OTC). Eles se posicionaram negativamente quanto a essa modalidade de venda, por acreditarem que a ampliação do acesso ao método poderia incentivar as pessoas a não se precaverem em suas relações sexuais, não utilizando o preservativo masculino ou outro método anticonceptivo. O raciocínio que compartilham sugere que quanto mais se amplia a disponibilidade do método, mais os indivíduos se tornariam negligentes ou displicentes com os cuidados necessários à prevenção da gravidez.

Alguns defendem um controle mais rigoroso nas vendas da contracepção de emergência nas farmácias, por meio da apresentação e/ou retenção de receita médica, como ocorre com outros medicamentos de uso controlado e antibióticos. Eles consideram que os riscos à saúde seriam ampliados caso o acesso ao medicamento fosse livre (OTC). Eles valorizam em suas narrativas o poder médico, destacando a legitimidade dos ginecologistas no que diz respeito à orientação e indicação de métodos contraceptivos. Justificam suas respostas afirmando que os médicos são os profissionais mais adequados para prestar qualquer esclarecimento quanto a esse medicamento, partindo do pressuposto de que há riscos em sua utilização, especialmente se usado de forma repetida.

Os balconistas não citaram o farmacêutico como uma alternativa de aconselhamento no que se refere ao uso de anticoncepcionais. Quando dizem que os "ginecologistas" deveriam orientar a usuária da contracepção de emergência, eles não identificam o farmacêutico como o profissional de saúde mais próximo ao cliente para transmitir informações confiáveis às consumidoras. Alguns autores argumentam que os profissionais do ramo farmacêutico, especialmente os farmacêuticos, estão em uma posição estratégica no que se refere à ampliação do acesso à contracepção de emergência, mas não possuem treinamento e estão muito envolvidos na lógica do mercado 33,34,35.

O debate internacional sobre a ampliação do acesso à contracepção de emergência traz à tona discussões acerca das atribuições e responsabilidades específicas das várias categorias profissionais nela envolvida e, até mesmo, sobre o grau de autonomia das mulheres e casais quanto às escolhas contraceptivas. Ao estudar o contexto de ampliação do acesso à contracepção de emergência na Austrália, Calabretto ${ }^{36}$ observa que a política de dispensação via farmácias gerou uma disputa política entre médicos e farmacêuticos. Percebe-se uma relação direta entre a noção de risco e a identificação da categoria médica como 
os profissionais que detêm a legitimidade para o aconselhamento das usuárias e prescrição do medicamento.

Indagados sobre os modos de interação com os clientes nessas ocasiões, os balconistas observam que o atendimento no balcão da farmácia não propicia um ambiente adequado para uma abordagem educativa sobre esse método contraceptivo. Salientam que o trabalho cotidiano de vendedor de medicamentos exercido pelo balconista não permite uma maior aproximação com os clientes, até mesmo pelo constrangimento e falta de privacidade para se tratar de assuntos íntimos. Por isso, eles reforçam a responsabilidade dos médicos, em primeiro lugar, e dos veículos de comunicação e mídia social e das escolas na tarefa de informar e esclarecer sobre o uso adequado do contraceptivo de emergência. Eles só oferecem orientação quando solicitada ou quando o conhecimento prévio com os clientes permite. Eximem-se assim de qualquer função educativa junto ao público que atendem nas farmácias.

As informações que eles possuem sobre esse anticoncepcional são, de modo geral, desatualizadas e pouco confiáveis. Elas são adquiridas com os próprios colegas nas farmácias, com representantes de laboratórios e coincidem com os questionamentos surgidos no balcão: o tempo para sua utilização e sua eficácia. Em relação a esses aspectos, a maioria informou que o tempo possível de uso após a relação sexual é de 72 horas; um deles disse 12 horas após o ato sexual. Vários afirmaram que a contracepção de emergência é $100 \%$ eficaz, mas que sua eficácia vai diminuindo com o tempo.

Nenhum dos pesquisados relacionou a contracepção de emergência a medicamentos abortivos, ao contrário de muitos estudos que demonstram grande abrangência desta representação, especialmente no contexto latino-americano 37,38,39,40. Como o contraceptivo de emergência é ingerido pós-coito, há um certo temor de que ele seja abortivo, prática ilegal no contexto brasileiro. Esse aspecto também esteve presente nas representações dos farmacêuticos, no âmbito da pesquisa mais ampla ( $\mathrm{n}=383$ ). Embora 71,5\% deles não a considerem abortiva, $89,9 \%$ a classificam como uma "bomba hormonal”. Nossa hipótese para tal deslocamento nas concepções sobre a contracepção de emergência sugere uma certa sofisticação dos argumentos contrários à sua aceitação, ressaltando-se mais a desregulação hormonal, a interrupção de um ciclo "natural" hormonal 41 do que propriamente supostos efeitos abortivos. Assim, a ênfase recai em um argumento considerado de ordem científica e não religiosa.
Por fim, quanto à experiência de uso pessoal da contracepção de emergência, dentre os entrevistados, sete deles já a haviam utilizado (cinco homens com suas namoradas/esposas e duas mulheres), em situações emergenciais e não corriqueiras ou banais, como eles distinguem. Justificaram sua utilização em contextos de relacionamentos afetivo-sexuais estáveis, em fase de amamentação da esposa ou de um "descuido", distinguindo-se das consumidoras as quais criticam por um uso repetitivo em relacionamentos ocasionais e fortuitos.

\section{Considerações finais}

Os balconistas entrevistados neste estudo, homens e mulheres, têm representações negativas sobre a contracepção de emergência. Em suas narrativas se destacam, sobretudo, opiniões relativas aos riscos advindos do uso "descontrolado" ou "indiscriminado" desse medicamento, especialmente por jovens adolescentes. Segundo a concepção que compartilham, trata-se de uma "bomba hormonal" que pode causar sérios danos, podendo levar à esterilização de mulheres jovens, ao câncer e outras doenças graves. Sobressai uma reprovação moral sobre o uso da contracepção de emergência porque ela evidencia uma falha anterior das mulheres em disciplinar seus corpos e exercício sexual, revelando estereótipos de gênero bem demarcados.

Tal como apontado em outras investigações, demonstram desconhecimento técnico em relação à contracepção de emergência, revelando a baixa propagação de informações confiáveis sobre o método. A precariedade das informações recebidas sobre o método, provenientes dos próprios colegas e dos laboratórios farmacêuticos, influencia as suas atitudes e reflexões sobre o método e sobre a utilização que os usuários (mulheres ou casais) fazem dele.

Há ausência de investimento público no Brasil em ações de capacitação dos profissionais do setor farmacêutico em questões relacionadas à saúde sexual e reprodutiva, para que possam relacionar a dispensa do medicamento com a oportunidade para informar às usuárias sobre a importância de utilização de um método anticoncepcional de uso regular, após o recurso à contracepção de emergência, direcionando-as aos serviços públicos de saúde.

Por um lado, o conhecimento precário que detêm sobre o método da contracepção de emergência, aliado ao fato de conferirem legitimidade somente aos médicos para orientar sobre o seu uso, excluindo os farmacêuticos, podem explicar o desconforto dos balconistas para abordar as 
usuárias quando se sentem incomodados pela constatação do uso repetitivo desse medicamento. Por outro lado, eles se sentem pressionados a somente vender o medicamento, sobretudo pela função que ocupam no comércio farmacêutico, conforme a lógica de mercado que domina o setor. A dimensão de cuidado à saúde estaria subsumida a tal lógica.

Por ser considerado um medicamento "perigoso", os balconistas preferem delegar a responsabilidade quanto à orientação e aconselhamento sobre o uso do método da contracepção de emergência aos médicos ginecologistas, os quais supostamente possuem conhecimento a respeito dos possíveis riscos associados ao uso de hormônios para os corpos femininos. Os pesquisados não avaliam que o farmacêutico possa ser um profissional de saúde importante e competente para orientar as usuárias sobre a contracepção de emergência em seu atendimento no balcão da drogaria.

Quanto à regulação sanitária da venda da contracepção de emergência, apresentam argumentos contrários à possibilidade de sua disponibilidade fora do balcão (OTC), o que facilitaria o livre acesso dos consumidores ao medicamento. Consideram que essa medida pode levar a um uso ainda mais "banalizado" do método, podendo também interferir de forma negativa e determinante na prevenção de DST/AIDS. Os entrevistados acreditam que a maior disponibilidade da contracepção de emergência encoraja práticas sexuais sem proteção, especialmente entre os jovens. Por isso, em geral, avaliam que deveria haver um maior controle sanitário da sua comercialização, tendo em vista que a prescrição médica exigida nunca é respeitada pelos consumidores, nem pelos estabelecimentos farmacêuticos privados.

Os resultados desta investigação recomendam a ampliação do debate sobre direitos sexuais e reprodutivos de homens e mulheres que precisam ter garantidos o acesso e a orientação para o uso da contracepção de emergência, em situações emergenciais, incluindo-se os farmacêuticos e balconistas de farmácias, além de profissionais de saúde e gestores públicos. As dificuldades do contexto brasileiro que se referem à incapacidade do SUS de ofertar cobertura gratuita, universal e acessível em tempo oportuno à contracepção de emergência, somada à vertiginosa expansão do comércio farmacêutico no país e às especificidades culturais da prática de automedicação entre nós, transformam tal desafio em algo premente.

\section{Colaboradores}

E. R. Brandão concebeu e coordenou a pesquisa, swendo responsável pela revisão e redação final do manuscrito. C. S. Cabral, M. Ventura, S. P. Paiva, L. L. Bastos, N. V. B. V. Oliveira e I. Szabo participaram de todas as etapas da pesquisa, colaborando na redação e revisão crítica do artigo.

\section{Agradecimentos}

À FAPERJ (processo no 110.085/2012) e CNPq (processo no 481422/2012-9) que apoiaram a pesquisa. 


\section{Referências}

1. Perpétuo IHO, Wong LLR. Desigualdade socioeconômica na utilização de métodos anticoncepcionais no Brasil: uma análise comparativa com base nas PNDS 1996 e 2006. In: Centro Brasileiro de Análise e Planejamento, Ministério da Saúde, organizador. Pesquisa Nacional de Demografia e Saúde da Criança e da Mulher - PNDS 2006: dimensões do processo reprodutivo e da saúde da criança. Brasília: Ministério da Saúde; 2009. p. 87-104.

2. Centro Brasileiro de Análise e Planejamento, Ministério da Saúde. Pesquisa Nacional de Demografia e Saúde da Criança e da Mulher. PNDS 2006 relatório final. Brasília: Ministério da Saúde; 2008.

3. Arrais PSD, Coelho HLL, Batista MCDS, Carvalho ML, Righi RE, Arnau JM. Perfil da automedicação no Brasil. Rev Saúde Pública 1997; 31:71-7.

4. Naves JOS, Castro LLC, Carvalho CMS, Merchán -Hamann E. Automedicação: uma abordagem qualitativa de suas motivações Ciênc Saúde Coletiva 2010; 15:1751-62.

5. Domingues PHF, Galvão TF, Andrade KRC, Sá PTT, Silva MT, Pereira MG. Prevalência da automedicação na população adulta do Brasil: revisão sistemática. Rev Saúde Pública 2015; 49:1-8.

6. Ministério da Saúde. Resolução RDC no 44, de 17 de agosto de 2009. Agência Nacional de Vigilância Sanitária dispõe sobre boas práticas farmacêuticas para o controle sanitário do funcionamento, da dispensação e da comercialização de produtos e da prestação de serviços farmacêuticos em farmácias e drogarias e dá outras providências. Diário Oficial da União 2009; 18 ago.

7. Oliveira AB, Oyakawa CN, Miguel MD, Zanin SMW, Montrucchio DP. Obstáculos da atenção farmacêutica no Brasil. RBCF Rev Bras Ciênc Farm 2005; 41:409-12.

8. Farina SS, Romano-Lieber NS. Atenção farmacêutica em farmácias e drogarias: existe um processo de mudança? Saúde Soc 2009; 18:7-18.

9. Paiva SP, Brandão ER. Contracepção de emergência no contexto das farmácias: revisão crítica de literatura. Physis (Rio J.) 2012; 22:17-34

10. Conrad P. The medicalization of society: on the transformation of human conditions into treatable disorders. Baltimore: Johns Hopkins University Press; 2007.
11. Williams SJ, Martin P, Gabe J. The pharmaceuticalisation of society? A framework for analysis. Sociol Health Illn 2011; 33:710-25.

12. Hardy E, Duarte GA, Osis MJD, Arce XE, Possan M. Anticoncepção de emergência no Brasil: facilitadores e barreiras. Cad Saúde Pública 2001; 17:1031-5.

13. Díaz S, Hardy E, Alvarado G, Ezcurra E. Acceptability of emergency contraception in Brazil, Chile, and Mexico. 1 - Perceptions of emergency oral contraceptives. Cad Saúde Pública 2003; 19: 1507-17.

14. Díaz S, Hardy E, Alvarado G, Ezcurra E. Acceptability of emergency contraception in Brazil, Chile, and Mexico. 2 - Facilitating factors versus obstacles. Cad Saúde Pública 2003; 19:1729-37.

15. Martin A. La anticoncepción de emergencia en América Latina y el Caribe. Rev Panam Salud Pública 2004; 16:424-31.

16. Simonds W, Ellertson C. Emergency contraception and morality: reflections of health care workers and clients. Soc Sci Med. 2004; 58:1285-97.

17. Pires AP. Amostragem e pesquisa qualitativa: ensaio teórico e metodológico. In: Poupart J, Deslauriers JP, Groulx LH, Laperriére A, Mayer R, Pires AP, organizadores. A pesquisa qualitativa: enfoques epistemológicos e metodológicos. Petrópolis: Editora Vozes; 2012. p. 154-211.

18. Paiva SP. Silêncio, não dito e vergonha no balcão da drogaria: estudo etnográfico sobre a comercialização da contracepção de emergência no Rio de Janeiro [Tese de Doutorado]. Rio de Janeiro: Instituto de Estudos em Saúde Coletiva, Universidade Federal do Rio de Janeiro; 2014.

19. Schutz A. Atenção seletiva: relevâncias e tipificação. In: Schutz A, organizador. Fenomenologia e relações sociais. Rio de Janeiro: Jorge Zahar Editor; 1979. p. 110-20.

20. Silverman D. Analysing talk and text. In: Denzin NK, Lincoln YS, editors. Handbook of qualitative research. 2nd Ed. Thousand Oaks: Sage Publications; 2000. p. 821-34.

21. Kornblit AL. Metodologías cualitativas en ciencias sociales: modelos y procedimentos de análisis. $2^{\mathrm{a}}$ Ed. Buenos Aires: Editorial Biblos; 2007.

22. Minayo MCS. Análise qualitativa: teoria, passos e fidedignidade. Ciênc Saúde Coletiva 2012; 17: 621-6 
23. Baldwin SB, Solorio MR, Washington DL, Yu H, Huang YC, Brown ER. Who is using emergency contraception? Awareness and use of emergency contraception among California women and teens. Womens Health Issues 2008; 18:360-8.

24. Rocca CH, Schwarz EB, Stewart FH, Darney PD, Raine TR, Harper CC. Beyond access: acceptability, use and nonuse of emergency contraception among young women. Am J Obstet Gynecol 2007; 196:29.e1-6.

25. Shoveller J, Chabot C, Soon JA, Levine M. Identifying barriers to emergency contraception use among young women from various sociocultural groups in British Columbia, Canada. Perspect Sex Reprod Health 2007; 39:13-20.

26. Hussainy SY, Stewart K, Chapman CB, Taft AJ, Amir LH, Hobbs MK, et al. Provision of the emergency contraceptive pill without prescription: attitudes and practices of pharmacists in Australia. Contraception 2011; 83:159-66.

27. Osis MJD, Faúndes A, Makuch MY, Mello MB, Souza MH, Araújo MJO. Atenção ao planejamento familiar no Brasil hoje: reflexões sobre os resultados de uma pesquisa. Cad Saúde Pública 2006; 22:2481-90.

28. Foucault M. História da sexualidade I: a vontade de saber. Rio de Janeiro: Edições Graal; 1999.

29. Teixeira M, Guillaume A, Ferrand M, Adjamabgo A, Bajos N. Representations and uses of emergency contraception in West Africa. A social anthropological reading of a northern medicinal product. Soc Sci Med 2012; 75:148-55.

30. Brigeiro M, Maksud I. Aparição do Viagra na cena pública brasileira: discursos sobre corpo, gênero e sexualidade na mídia. Revista Estudos Feministas 2009; 17:71-88.

31. Faro L, Chazan LK, Rohden F, Russo J. Homem com "H": ideais de masculinidade (re)construídos no marketing farmacêutico. Cadernos Pagu 2013; 40:287-321.

32. Rovira PS. La Viagra nuestra de cada día. Consumo recreacional y angustias masculinas con respecto a su potencia eréctil. Sex Salud Soc (Rio J.) 2014; (18):140-60.
33. Agomo CO. The role of community pharmacists in public health: a scoping review of the literature. J Pharm Health Serv Res 2012; 3:25-33.

34. Habel MA, Leichliter JS. Emergency contraception and risk for sexually transmitted infections among U.S. women. J Womens Health (Larchmt) 2012; 21:910-6.

35. Moreau C, Trussell J, Glasier A. Challenging the "insurance theory" of sexual and reproductive health behaviors. Contraception 2012; 85:9-10.

36. Calabretto H. Australia: organized physician opposition to nonprescription status. In: Foster A, Wynn LL, editors. Emergency contraception: the story of a global reproductive health technology. New York: Palgrave Macmillan; 2012. p. 207-20.

37. Cooper R, Bissell P, Wingfield J. Ethical, religious and factual beliefs about the supply of emergency hormonal contraception by UK community pharmacists. J Fam Plann Reprod Health Care 2008; 34:47-50.

38. Pecheny M, Tamburrino MC. $i$ “La palabra lo dice”? Interpretaciones cruzadas y obstáculos al acceso a la anticoncepción de emergencia. Sex Salud Soc (Rio J.) 2009; (1):158-76.

39. Richman AR, Daley EM, Baldwin J, Kromrey J, O'Rourke K, Perrin K. The role of pharmacists and emergency contraception: are pharmacists' perceptions of emergency contraception predictive of their dispensing practices? Contraception 2012; 86:370-5.

40. Bergallo P. El debate jurídico en torno a la anticoncepción de emergencia: una mirada comparada. In: Arilha M, Lapa TS, Pisaneschi TC, organizadores. Contracepção de emergência no Brasil e América Latina: dinâmicas políticas e direitos sexuais e reprodutivos. São Paulo: Oficina Editorial; 2010. p. 9-62.

41. Oudshoorn N. Beyond the natural body: an archeology of sex hormones. London: Routledge; 1994. 


\section{Abstract}

This study focused on views towards emergency contraception among pharmacy attendants in Greater Metropolitan Rio de Janeiro, Brazil. The empirical material came from a socio-anthropological study with 20 semi-structured interviews of pharmacy attendants of both sexes (8 females and 12 males). The interviews showed negative views of emergency contraception emphasizing its potential health risks. Interviews considered emergency contraception a "hormone bomb" that can harm the female reproductive organs and other organ systems. The pharmacy attendants high lighted the risks of "uncontrolled" or "indiscriminate" use, especially by adolescents and young women. Since they considered it "dangerous" to women's bodies, they assigned the responsibility for orientation and counseling on use of the method to gynecologists rather than to pharmacists. The article discusses the need to expand the public debate on emergency contraception in Brazil to include pharmacists and pharmacy attendants, in addition to health professionals in general and teachers.

Postcoital Contraception; Pharmaceutical Care; Sexuality; Reproductive Health

\section{Resumen}

La investigación tuvo como objetivo conocer la perspectiva de los auxiliares de farmacias sobre los contraceptivos de emergencia en la Región Metropolitana de Río de Janeiro, Brasil. El material empírico proviene de una investigación socioantropológica con 20 entrevistas semiestructuradas con auxiliares de sexo femenino (8) y masculino (12). Los entrevistados presentan concepciones negativas sobre la anticoncepción de emergencia, enfatizando los riesgos que puede provocar en la salud. El medicamento es considerado una "bomba hormonal" que puede causar daños a los órganos reproductivos femeninos y otros sistemas del cuerpo. Ellos destacan los riesgos del uso "descontrolado" o "indiscriminado", especialmente por adolescentes y mujeres jóvenes. Por ser considerado "peligroso" para el cuerpo femenino, ellos atribuyen la responsabilidad de la orientación y asesoría sobre el uso de este método anticonceptivo a los médicos ginecólogos, y no a los farmacéuticos. Se discute la necesidad de la ampliación del debate público sobre la anticoncepción de emergencia en Brasil, incluyéndose a farmacéuticos y auxiliares de farmacia, además de los profesionales de salud y educadores.

Anticoncepción Poscoital; Atención Farmacéutica; Sexualidad; Salud Reproductiva
Recebido em 21/Ago/2015

Versão final reapresentada em 15/Jan/2016

Aprovado em 03/Fev/2016 